\title{
Solid State Polymorphism and Dynamics of 2,2-Dimethylbutan-1-ol as Studied by Adiabatic Calorimetry and Dielectric Spectroscopy
}

\author{
E. Juszyńska-Ga€ĄzKa, P.M. Zieliński, M. Massalska-Arodź And J. Krawczyk \\ The H. Niewodniczański Institute of Nuclear Physics, PAS, Kraków, Poland
}

\begin{abstract}
2,2-dimethylbutan-1-ol, known as neohexanol, was studied by adiabatic calorimetry and dielectric spectroscopy. Details of complex solid state polymorphism and relaxational dynamics were identified and described. System of phases of neohexanol was found to be monotropic with three orientationally disordered crystalline phases besides isotropic liquid and ordered crystal. Moreover, two subsequent glass transition anomalies of heat capacity $C_{p}(T)$ were detected on heating in one of the supercooled orientationally disordered crystalline phases. Thermodynamical properties of neohexanol are described in relation to the dielectric relaxation processes found.
\end{abstract}

DOI: 10.12693/APhysPolA.124.917

PACS: 65.60.+a, 77.22.Gm, 64.70.P-

\section{Introduction}

In an ordered crystalline phase, molecules are arranged in a well-defined crystal lattice. Their positional and orientational degrees of freedom are activated at melting. In many compounds of non-rigid organic molecules the process of activation of various degrees of freedom occurs by stages of several subsequent phase transitions below the temperature range of the isotropic phase. In some isotropic liquids a tendency to glass formation is larger than to crystallization [1].

At glass transition, dynamical degrees of freedom are frozen out, leaving the molecules at random positions and/or orientations. Signatures of glass transition depend on the experimental method applied. In heat capacity measurement such a transition shows as a pronounced jump of $C(T)$ (a weak second order transition), well below the melting point of a crystalline phase. In the dielectric relaxation method glass transition of liquid phase manifests itself by dramatic slowing down of relaxation time $\tau-$ it is assumed that temperature $T=T_{\mathrm{g}}$ when $\tau(T) \cong 100 \mathrm{~s}$. The fact that also a supercooled mesophase, with partial long range order, can be a glass-former is known since 1968 [2] for rotatory phases and since early seventies for anisotropic liquid crystals [3]. The observed solid state polymorphism results from an interplay of several factors such as structure of the molecules, their interactions and dynamics. Since the role of molecular conformations in the process of formation of a particular solid phase is crucial, the studies of various isomers should help to understand this process. It is well known that isomers of cyanobiphenyls exhibit different phase diagrams: substances with chiral molecules form glass of cholesteric phase, while non-chiral molecules exhibit a sequence of liquid crystalline phases and a crystal state [4].

It is also valid for isomers of neohexanol, where systematic differential scanning calorimetry (DSC) studies showed various sequences of mesophases [5]. It is worth to note that various isomers of neohexanol have different number of conformations. For neohexanol there are three: trans, gauche+ and gauche-. In the IR studies dependence of the strength of hydrogen bonds on localization of the $\mathrm{OH}$ groups in isomeric molecules was observed [5-7]. Additionally, at melting some thermodynamical parameters are different for various isomers as calculated using extended Landau theory [8].

The present paper is aimed to elucidate the complexity of solid state polymorphism of neohexanol compound in detail by measuring the heat capacity in adiabatic conditions and the complex dielectric permittivity after well-defined thermal treatment of the sample, taking into account the literature data available so far $[5-20]$. Research into the nature of solid phases of the neohexanol is a part of a larger project involving studies of thermodynamic and dynamic properties of the isomeric dimethylbutanols [5-14] and also neoalcohols of similar chemical structure of molecules.

Neohexanol, abbreviated as 2,2-DM-1-B, with chemical formula $\mathrm{CH}_{3} \mathrm{CH}_{2} \mathrm{C}\left(\mathrm{CH}_{3}\right)_{2} \mathrm{CH}_{2} \mathrm{OH}$, is one of the isomers of dimethylbutanols. Their branched alcohol molecules have globular shapes, what facilitates solid state polymorphism with orientationally disordered crystals (ODIC). On slow cooling, the glass of isotropic liquid was obtained in one isomer $(3,3-\mathrm{DM}-1-\mathrm{B})[6,10,13]$ and the glass of ODIC phase in two other (3,3-DM-2-B and 2,2-DM-1-B). The 2,3-DM-2-B isomer was proved to crystallise. The phase diagram of neohexanol is still not clear despite the studies performed with differential thermal analysis (DT) (under ambient and elevated pressure) $[15,16]$, DSC $[5,6,17]$, dielectric relaxation $[6,10$, 15-20], and inelastic incoherent neutron scattering $[6,7$, $9,11-13]$ methods. The DT results published by Chan and Johari [18, 19] showed a crystallization at $183 \mathrm{~K}$ and melting at $241 \mathrm{~K}$. In DT studies of Edelmann [15, 16] five solid phases (with additional one at higher pressure) were found in the following phase transitions: $\mathrm{C}_{\mathrm{III}}{ }^{\prime \prime} \rightarrow \mathrm{C}_{\mathrm{II}}$ 
$(187.9 \mathrm{~K}), \mathrm{C}_{\mathrm{III}^{\prime}} \rightarrow \mathrm{C}_{\mathrm{II}}(196.4 \mathrm{~K}) \mathrm{C}_{\mathrm{III}} \rightarrow \mathrm{C}_{\mathrm{II}}(198.6 \mathrm{~K})$, $\mathrm{C}_{\mathrm{II}} \rightarrow \mathrm{C}_{\mathrm{I}}(210.4 \mathrm{~K}), \mathrm{C}_{\mathrm{I}} \rightarrow \mathrm{I}(250.6 \mathrm{~K})$ observed on heating, and a glass transition of disordered crystal $\mathrm{C}_{\mathrm{II}}$ at $T_{\mathrm{g}}=157.8 \mathrm{~K}$.

Dworkin's DSC measurement showed a glass transition at $147 \mathrm{~K}$, a spontaneous crystallization anomaly at $180 \mathrm{~K}$, and melting at $224.5 \mathrm{~K}$ (onset value) [17]. In our DSC measurements [5] a previously nonobserved phase transition anomaly at about $235 \mathrm{~K}$ was found (see Fig. 1): when the sample was cooled down to $150 \mathrm{~K}$ from $300 \mathrm{~K}$ at $-10 \mathrm{~K} / \mathrm{min}$, a broad peak of complex crystallization was detected at $212 \mathrm{~K}$ (the peak position value), while on heating an endothermic transition at $234.7 \mathrm{~K}$ and melting at $T_{\mathrm{m}}=251 \mathrm{~K}$ were registered [5]. However, the sample abruptly cooled down to $100 \mathrm{~K}$, and then heated, crystallised at $180 \mathrm{~K}$ and melted at $235.5 \mathrm{~K}$. Dielectric studies did not show a consistent picture of phase sequence either. Dielectric relaxations $\alpha$ and $\beta$ were detected for liquid and plastic crystal phases, respectively, by Chan and Johari $[18,19]$ in the frequency range between $100 \mathrm{~Hz}$ and $10^{5} \mathrm{~Hz}$.

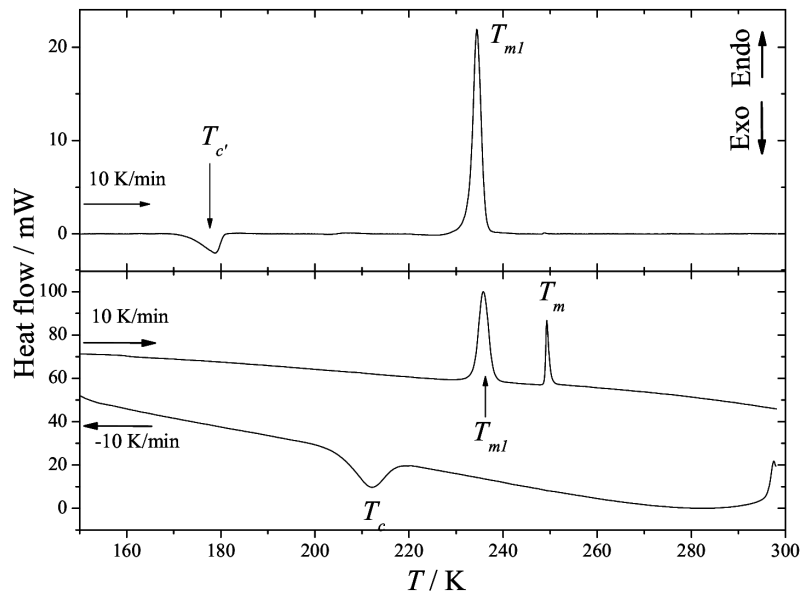

Fig. 1. DSC thermograms of 2,2-DM-1-B obtained with the scanning rate of $10 \mathrm{~K} / \mathrm{min}$ [5]: on heating after fast cooling of the sample (top part), and on cooling (bottom curve) and heating (upper curve) in bottom part. $T_{\mathrm{c}^{\prime}}$ and $T_{\mathrm{c}}$ refer to two crystallization processes.

A glass transition at $163 \mathrm{~K}$ was mentioned by Dannhauser and co-workers [20]. They studied dielectric relaxation in liquid phase to about $240 \mathrm{~K}$ and in rotatory phase down to $200 \mathrm{~K}$. Edelmann $[15,16]$ observed a dielectric relaxation in the liquid phase supercooled down to $213 \mathrm{~K}$, at which point it turned into crystal. The latter then melted between $243 \mathrm{~K}$ and $253 \mathrm{~K}$. Neutron powder diffraction experiment [9] allowed us to differentiate between phases: for ordered phase named $C 3$ several Bragg reflections were observed and for glass of ODIC phase, denoted as $C 2$, no reflections were found. However, no structure identification was possible, as neutron diffraction on a hydrogen-rich molecular crystal becomes obscured by a very large incoherent background. X-ray diffraction was not successful, either. Glass of $C 2$ ob- tained on fast cooling was identified also by non-Debye behavior of density of states at about $30 \mathrm{~cm}^{-1}$, forming the so-called boson peak. On heating, after transition of this glass to phase $C 2$, the well-ordered crystalline phase $C 3$ and then again $C 2$ were obtained. Reorientations of molecules in phase $C 2$ were evidenced by observation of the broadening of the peak of elastic scattering [9].

\section{Experimental}

\subsection{Calorimetric measurements}

The 2,2-DM-1-B compound was synthesized in the Institute of Pharmacology, PAS, Kraków. The heat capacity $C_{p}(T)$ of 2,2-DM-1-B was measured by means of a home-made high-accuracy adiabatic calorimeter at the Division of Condensed Matter Physics, IFJ PAN in Kraków. The liquid sample of $5.5279 \mathrm{~g}(54.1024 \mathrm{mmol})$ was enclosed in the cylindrical calorimeter cell made of gold-plated copper. Temperature dependence of heat capacity was measured on heating using the adiabatic regime (so-called intermittent heating method or point method) in the temperature range from liquid nitrogen temperature to $300 \mathrm{~K}$, in several runs after slow and fast cooling of the sample.

In order to achieve good accuracy of the measured thermal effects the heat exchange between the cell and the environment has to be diminished. The sample container was hung on a silk thread inside a pair of coaxial thermal shields equipped with heaters and enclosed in a vacuum-tight steel vessel evacuated down to $c a \cdot 10^{-5}$ mbar. The system was housed in a liquid nitrogen cryostat. The temperature was measured with the accuracy of $10 \mu \mathrm{K}$ using a platinum thermometer. In addition, to ensure adiabatic conditions, several two-point thermocouples located between the cell and the thermal shields were used to control heat exchange in the system. The on-line computer with the dedicated software [21] was used to control parameters of the experiment and to acquire the data.

In this adiabatic mode the temperature of the sample cell is kept constant under adiabatic condition through strict control of temperature of both the inner and the outer adiabatic shields. The temperature of the sample cell is constantly measured and when it reaches equilibrium, a well-defined portion of energy is introduced to the system by means of electric current applied for constant period of time to the heater located in the core of the cell.

Following the heating period, the temperature of the sample cell varies due to temperature gradient in the system of sample cell and adiabatic shields. This temperature gradient originates from a continuous flow of the introduced energy, being distributed within the sample volume, and the heat exchange between sample cell and thermal shields. However, after certain period of time such temperature variation, owing to the first source (introduced heat), will disappear while the second one, related to non-ideal adiabatic conditions, will become stable (assuming constant temperatures of the inner and the outer adiabatic shields). Thus, the relation between the 
temperature of the sample cell and time will become linear. This feature can be utilized to determine whether the sample has already reached the thermal equilibrium. Namely, if the variation of the sample cell temperature measured after the heating period becomes sufficiently small (e.g. $0.001 \mathrm{~K} / \mathrm{min}$ ), the system can be regarded as having reached thermal equilibrium. Then a next heat pulse can be applied to the sample.

To investigate the phase situation on cooling the sample as well as on heating, the instrument was operated in a pseudo-DT regime (the so-called "drift method"). Time evolution of temperature $T$ along with the rate of temperature changes, $\mathrm{d} T / \mathrm{d} t$, were registered during continuous cooling or heating of the sample by means of keeping the inner thermal shields at the temperature below or above that of the sample cell, respectively. Such a regime of measurement enabled us to perform a faster, as compared to adiabatic mode, but only qualitative observation of spontaneous thermal effects in the sample and to estimate the phase transition temperatures.

\subsection{Dielectric measurements}

The dielectric measurements of the complex electric permittivity, $\varepsilon^{*}(\nu)=\varepsilon^{\prime}(\nu)-\mathrm{i} \varepsilon^{\prime \prime}(\nu)$, were performed in the frequency range from $0.03 \mathrm{~Hz}$ to $1 \mathrm{MHz}$ using the Solartron-Schlumberger frequency response analyzer FR 1260 with the Novocontrol Spectrometer. The $\varepsilon^{\prime}$ and $\varepsilon^{\prime \prime}$ values were measured with accuracy better than $1 \%$ and $2 \%$, respectively. Measurements covered the temperature range from $123 \mathrm{~K}$ to $300 \mathrm{~K}$. The measurement run for each temperature was started at the higher frequency side and lasted for about $10 \mathrm{~min}$. The sample was kept between two brass disc-shaped electrodes. The diameter of the cell was $30 \mathrm{~mm}$ while the distance between the electrodes was $0.10 \mathrm{~mm}$. Separation between the electrodes was maintained by PTFE spacers. Measurements were performed at $2-10 \mathrm{~K}$ intervals on slow cooling of the sample and also on heating in two experimental regimes, i.e., after slow and after fast cooling. The temperature of the sample was controlled using Novocontrol Quatro system with a liquid nitrogen cryostat and measured with the accuracy of $0.1 \mathrm{~K}$.

\subsection{Purity determination}

The molar purity of the neohexanol used was found to be $99.67 \%$. It was calculated using the van't Hoff law [22]:

$$
T_{\text {triple }}-T_{\text {eq }}=\frac{R T_{\text {triple }}^{2}}{\Delta H_{\text {fus }}} \frac{x}{F},
$$

from the so-called melting fraction experiment performed by adiabatic calorimetry (see Fig. 2). The fully melted state corresponds to $F=1$. $T_{\text {triple }}$ is the triple point temperature, $T_{\mathrm{eq}}$ is the experimental equilibrium temperature of the fusion in the melted fraction $F$ of the substance, $R$ is the gas constant, $x$ means molar fraction of impurity and $\Delta H_{\text {fus }}$ is the calculated molar enthalpy of fusion [22]. The triple point temperature of $233.19 \mathrm{~K}$ was obtained by extrapolation of the slope of $T(1 / F)$ to the value of $1 / F=0$.

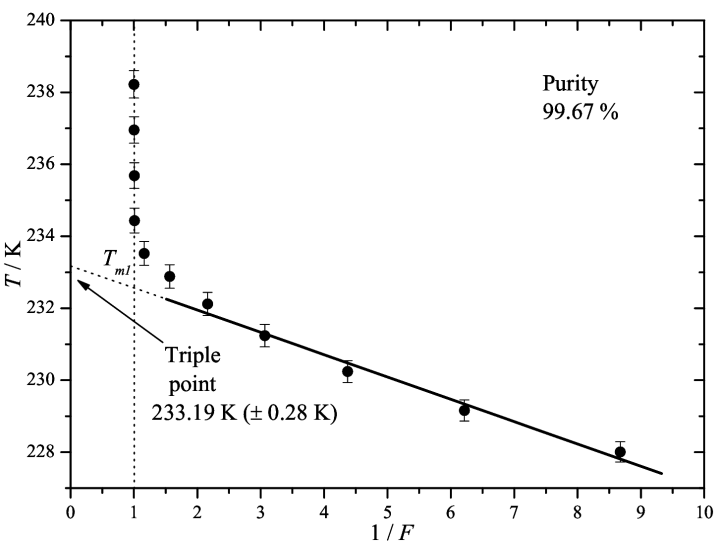

Fig. 2. Equilibrium temperature in the melt of neohexanol vs. inverse of the melted fraction $F$. The linear part of the $T(1 / F)$ plot indicates that the impurity does form an eutectic system with the substance. Error bars were calculated by standard deviation.

\section{Results}

\subsection{Phase transitions in neohexanol}

The temperature dependence of heat capacity $C_{p}(T)$ of neohexanol measured in adiabatic mode on heating of quenched (empty circles) and of slowly cooled (filled circles) sample from the liquid phase down to $100 \mathrm{~K}$ are presented in Fig. 3. All the $C_{p}(T)$ values were registered in equilibrium conditions. In temperature range between $100 \mathrm{~K}$ and $180 \mathrm{~K}$ the $C_{p}(T)$ depends on the way of cooling of the sample. Above $180 \mathrm{~K}$ the same sequence of three phase transitions was found. On slow cooling of the sample, a crystal phase, labelled $C 3$ was obtained, as reported in our previous paper [5].

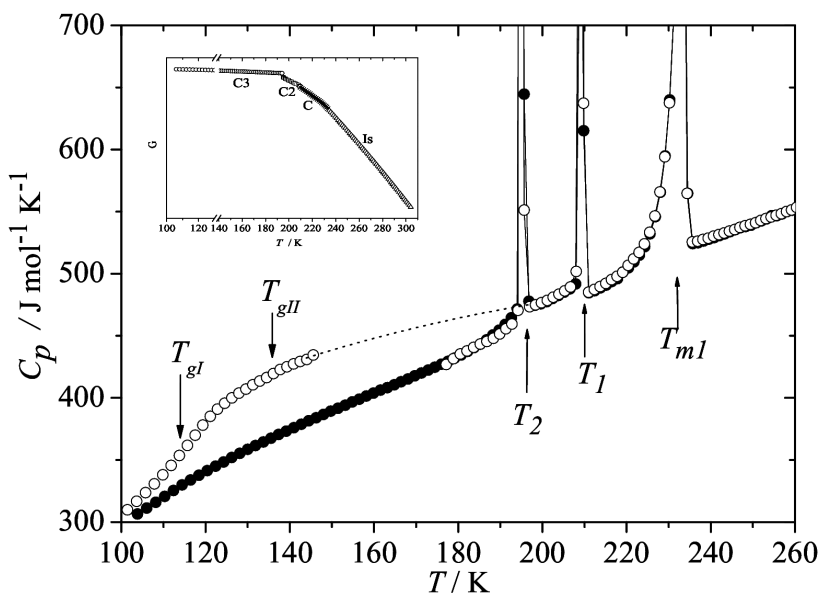

Fig. 3. Heat capacity $C_{p}$ of neohexanol vs. temperature measured for heating after slow (filled circles) and fast (open circles) cooling of the sample. Inset shows free energy vs. temperature for 2,2-DM-1-B, estimated basing on $C_{p}(T)$ measurements performed after slow cooling.

Anomalies (observed on heating) at $T_{2}=194.9 \mathrm{~K}$ and $T_{1}=209.2 \mathrm{~K}$ are solid-solid transitions, while 
the last one at $T_{\mathrm{m} 1}=233.6 \mathrm{~K}$ is related to melting of phase denoted here as $C$, and found in our DSC measurements (see Fig. 1) [5]. We detected the following phase sequence: $C 3(194.5 \mathrm{~K}) \rightarrow C 2$ $(209.2 \mathrm{~K}) \rightarrow C(233.6 \mathrm{~K}) \rightarrow s I L$, where $s I L$ means supercooled isotropic phase. No further anomaly was observed till room temperature despite several trials to find melting at about $250 \mathrm{~K}$. The enthalpy and entropy gained at the phase transitions $C 3 \rightarrow C 2$; $C 2 \rightarrow C$ and $C \rightarrow s I L$ were estimated to be $\Delta H=5772.6 \mathrm{~J} / \mathrm{mol} ; \Delta S=29.6 \mathrm{~J} /(\mathrm{mol} \mathrm{K}) ; \Delta H=$ $2689.1 \mathrm{~J} / \mathrm{mol} ; \Delta S=12.8 \mathrm{~J} /(\mathrm{mol} \mathrm{K})$ and $\Delta H=$ $4415 \mathrm{~J} / \mathrm{mol} ; \Delta S=17.9 \mathrm{~J} /(\mathrm{mol} \mathrm{K})$, respectively. The largest changes of enthalpy and entropy values were observed for the $C 3 \rightarrow C 2$ transition which points to activation of rotational motions in the ODIC $C 2$ phase. The entropy change at melting of phase $C$ is smaller than $21 \mathrm{~J} /(\mathrm{mol} \mathrm{K})$. According to the Timmermans criterion [23] it means that during melting of phase $C$ only translational degrees of freedom become active. In the $C 2 \rightarrow C$ transition the entropy change is the smallest and it most likely corresponds to the difference between structures of the $C 2$ and $C$ ODIC phases. Inset in Fig. 3 shows the Gibbs free energy vs. $T$ calculated from experimental data of the specific heat (filled circles). Choosing the reference state at $0 \mathrm{~K}$ required extrapolating the temperature dependence of the specific heat to this point. Cubic polynomial $C_{p}(T)=B T+C T^{3}$ was fitted in the whole temperature range of the crystalline $C 3$ phase. The heat capacity below $100 \mathrm{~K}$ was approximated by $C_{p}($ crystal $)=2.34328 T+0.00001 T^{3}$ and $C_{p}$ (glass) $=3.1558 T+8.82 \times 10^{-17} T^{3}$, where the $T^{3}$ term represents the Debye contribution.

Fast cooling of the sample leads to $C_{p}(T)$ values much higher than those for $C 3$ phase observed on heating up to $146 \mathrm{~K}$ as shown in Fig. 3 (open circles). In view of our earlier neutron measurements the corresponding phase was identified as supercooled disordered crystal $C 2$ [9]. Careful examination of the data in this temperature range reveals two anomalies, which can be ascribed to subsequent glass transitions at $T_{\mathrm{gI}} \approx 123 \mathrm{~K}\left(\Delta C_{p}=\right.$ $19 \mathrm{~J} /(\mathrm{mol} \mathrm{K})$ ) and at $T_{\mathrm{gII}} \approx 137 \mathrm{~K}$ (much smaller $\Delta C_{p}$ value). During further heating above $146 \mathrm{~K}$, several spontaneous transformations to metastable phases occurred (in Fig. 3 there are no $C_{p}(T)$ experimental points up to $180 \mathrm{~K}$ due to the lack of the thermodynamic equilibrium). Such metastable thermodynamic states were also detected by the earlier DT studies $[15,16]$. At $180 \mathrm{~K}$ a stabilisation to the ordered phase $C 3$ was observed. During cooling this phase down to $120 \mathrm{~K}$ no extra phase transition was detected. The values of the phase transition temperatures observed above $180 \mathrm{~K}$ were very close to those obtained during experimental runs performed after cooling the sample with various rates.

Also, the $C_{p}(T)$ anomaly observed on melting of phase $C$, and corresponding to the activation of translational motions, did not depend on the cooling rate. However, in phase $C 3$ observed after a series of metastable transitions for rapidly cooled sample the $C_{p}(T)$ values (o) were slightly lower than those registered after slow cooling $(\bullet)$. Moreover, the magnitudes of the thermal effects were sensitive to the regime of the measurement applied. The intensity of anomaly of $C_{p}(T)$ at about $195 \mathrm{~K}$, ascribed to the phase transition $C 3 \rightarrow C 2$, was bigger when measured after fast cooling of the sample than after a slower one. The opposite behavior was registered for the intensity of anomaly at $209.2 \mathrm{~K}$, ascribed to the $C 2 \rightarrow C$ transition. These effects mean that the pure phase $C 3$ was created at $180 \mathrm{~K}$ on heating only for a sample cooled rapidly just before the heating run. Temperatures of all phase transitions found in our calorimetric measurements are collected in Table I.

TABLE I

Details of various phase transitions obtained by adiabatic calorimetry (AC), the drift method, dielectric spectroscopy (DS) and inelastic incoherent neutron scattering (IINS) methods.

\begin{tabular}{|c|c|c|}
\hline Phase transition & Transition details & $\begin{array}{l}\text { Experimental } \\
\text { method }\end{array}$ \\
\hline \multicolumn{3}{|l|}{ On slow cooling } \\
\hline$I L-C 1$ & $251 \mathrm{~K}$ & DS \\
\hline$s I L-C 1$ & $226.6 \mathrm{~K}$ & drift \\
\hline$s I L-C$ & $223.2 \mathrm{~K}$ & \\
\hline$s C 1-C 2$ & $199 \mathrm{~K}$ & \\
\hline$(s C 2+s C)-C 3$ & $183.4 \mathrm{~K}$ & \\
\hline$I L-C 1+C$ & $212 \mathrm{~K}$ & DSC [3] \\
\hline $\begin{array}{c}\text { on fast cooling } \\
I L-C 2\end{array}$ & $181 \mathrm{~K}$ & drift \\
\hline \multicolumn{3}{|l|}{ On heating } \\
\hline $\begin{array}{l}\text { two glass } \\
\text { transitions }\end{array}$ & $\begin{array}{c}123 \mathrm{~K} \text { (after fast cooling) } \\
\Delta C_{p}=19 \mathrm{~J} /(\mathrm{K} \mathrm{mol}) \\
137 \mathrm{~K}(\text { near } 140 \mathrm{~K})\end{array}$ & $\begin{array}{c}\mathrm{AC} \\
\mathrm{AC}(\mathrm{DS})\end{array}$ \\
\hline $\begin{array}{c}s C 2-C 3 \\
\text { in several } \\
\text { non-equilibrium } \\
\text { transformations }\end{array}$ & $\begin{array}{c}\text { (after fast cooling) } \\
146-180 \mathrm{~K} \\
(153-183 \mathrm{~K}) \\
\text { below } 200 \mathrm{~K}\end{array}$ & $\begin{array}{c}\text { AC } \\
\text { DS } \\
\text { IINS [4] }\end{array}$ \\
\hline$C 3-C 2$ & $\begin{array}{c}195 \mathrm{~K} \\
\Delta H=5772.6, \Delta S=29.6 \\
\text { about } 210 \mathrm{~K}\end{array}$ & $\begin{array}{c}\mathrm{AC} \\
\text { IINS [4] }\end{array}$ \\
\hline $\begin{array}{l}C 2-C \\
C 3-C\end{array}$ & $\begin{array}{c}209.2 \mathrm{~K} \\
\Delta H=2689.1, \Delta S=12.8 \\
188-213 \mathrm{~K}\end{array}$ & $\mathrm{AC}$ \\
\hline$C-s I L$ & $\begin{array}{c}233.6 \mathrm{~K}(233 \mathrm{~K}) \\
\Delta H=4415, \Delta S=17.9 \\
235.5 \mathrm{~K} \text { (after fast cooling) } \\
\Delta H=847, \Delta S=3.6\end{array}$ & $\begin{array}{l}\mathrm{AC}(\mathrm{DS}) \\
\mathrm{DSC}[3]\end{array}$ \\
\hline$C 1-I L$ & $\begin{array}{c}250.8 \mathrm{~K} \\
\Delta H=585.5, \Delta S=2.3 \\
\text { near } 251 \mathrm{~K}\end{array}$ & $\begin{array}{c}\text { DSC [3] } \\
\text { DS }\end{array}$ \\
\hline
\end{tabular}

\subsection{Dynamics of neohexanol molecules}

The dielectric spectra registered on slow cooling of the sample down to $203 \mathrm{~K}$ with the rate of about $1 \mathrm{~K} / \mathrm{min}$ give the evidence of crystallization of the liquid phase in the temperature range between $253 \mathrm{~K}$ and $243 \mathrm{~K}$ to a rotatory phase. This phase is denoted here as $C 1$, because on subsequent heating, it melted to the liquid phase $I L$ between $243 \mathrm{~K}$ and $251 \mathrm{~K}$, as is shown in Fig. 4 . The 


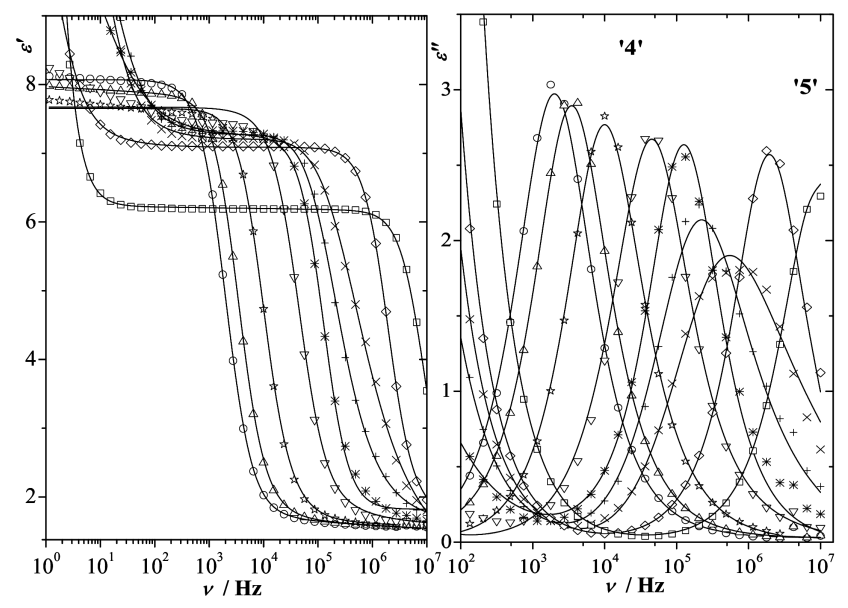

Fig. 4. Dielectric dispersion (left) and loss (right) data for 2,2-DM-1-B observed on slow heating, showing two relaxation processes: "4" in crystal $C 1$ at $211 \mathrm{~K}(\circ)$, $215 \mathrm{~K}(\triangle), 223 \mathrm{~K}(*), 235 \mathrm{~K}(\nabla)$ and $243 \mathrm{~K}(*)$ and "5" in the liquid at $251 \mathrm{~K}(\diamond)$ and $263 \mathrm{~K}(\square)$. At $247 \mathrm{~K}(+)$ and $249 \mathrm{~K}(\times)$ melting occurred. The solid lines are the Havriliak-Negami fits.

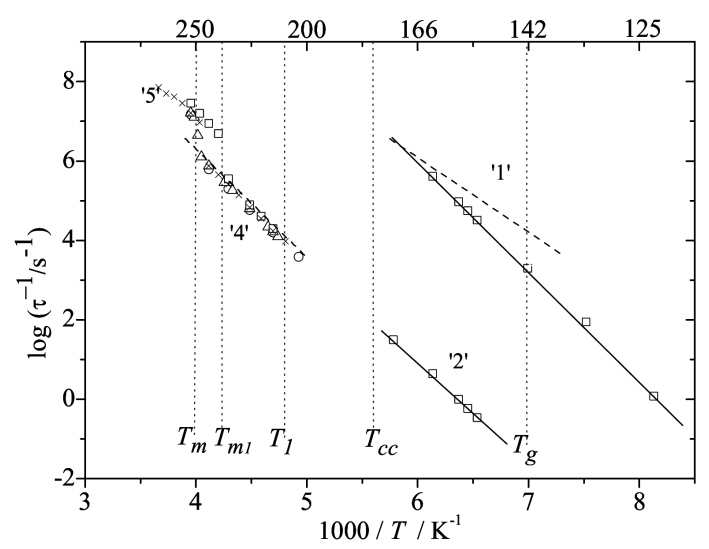

Fig. 5. Temperature dependence of the relaxation rates $\lg \left(\tau^{-1} / \mathrm{s}^{-1}\right)$ for 2,2-DM-1-B for three regimes of measurements: on heating after fast cooling (squares), on slow cooling (circles) and heating (triangles). The dashed lines are the literature data from [19, 20] and crosses from $[17,18]$. The vertical dotted lines show the approximated temperatures of phase transitions.

$C 1 \leftrightarrow I L$ transition, not observed in adiabatic calorimetry, is well illustrated by changes of relaxation time $\tau(T)$ (see Fig. 5). In order to describe the relaxation process in $C 1$ and $I L$ phases, the Havriliak-Negami formula $[24,25]$ :

$$
\varepsilon^{*}(\nu)=\varepsilon_{\infty}+\Delta \varepsilon /\left[1+(\mathrm{i} \omega \tau)^{1-\alpha}\right]^{\beta}+/\left(\nu-\nu_{0}\right),
$$

was fitted to the data (see solid lines in Fig. 4). The relaxation time $\tau=(1 / 2) \pi \nu_{\max }(T)$, where $\nu_{\max }$ is the frequency at the maximum of the absorption curve and correlation parameters $\alpha$ and $\beta$ fulfill the relation $0<(1-\alpha), \beta(1-\alpha) \leq 1$. For $\beta(1-\alpha)=(1-\alpha)=1$ the process is of the Debye type. The values of $\alpha$ and $1-\beta$ can be regarded as a measure of the local and long range

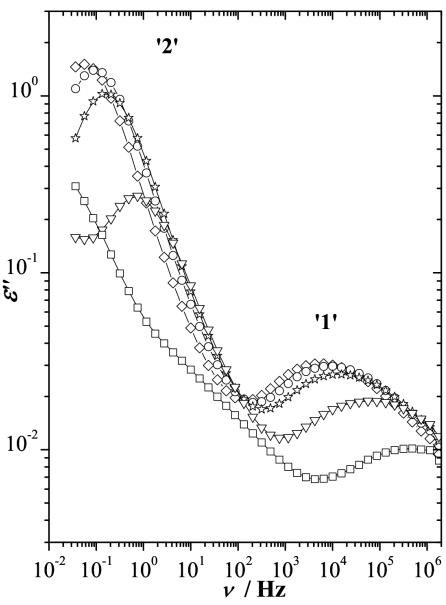

Fig. 6. Dielectric absorption curves $\log \varepsilon^{\prime \prime}(\log \nu)$ for the fast relaxation process " 1 " and slower relaxation process "2" for 2,2-DM-1-B in the temperature range from $153 \mathrm{~K}$ to $173 \mathrm{~K}$ observed on heating after fast cooling. Meaning of symbols: $153 \mathrm{~K}(\diamond), 155 \mathrm{~K}(\circ), 157 \mathrm{~K}(*)$, $163 \mathrm{~K}(\nabla), 173 \mathrm{~K}(\square)$. The solid lines are the HavriliakNegami fits.

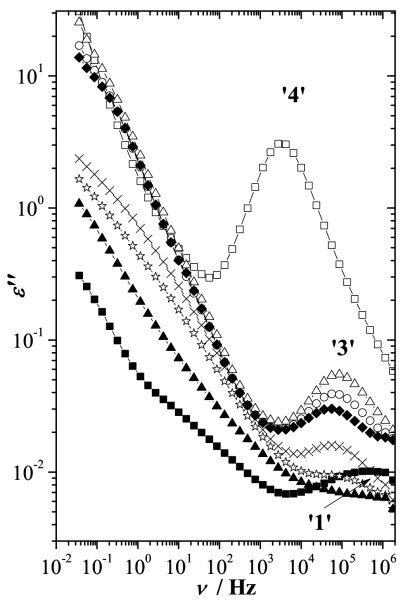

Fig. 7. Dielectric loss data for 2,2-DM-1-B showing process " 1 " at $173 \mathrm{~K}(\mathbf{-})$, process " 3 " for $188 \mathrm{~K}$ (*), $193 \mathrm{~K}(\times), 198 \mathrm{~K}(\bullet), 203 \mathrm{~K}(\triangle)$ and $208 \mathrm{~K}(\square)$ and process "4" at $213 \mathrm{~K}(\square)$. At $183 \mathrm{~K}(\mathbf{\Delta})$ no dynamics is visible. The solid lines are the Havriliak-Negami fits.

correlations for the relaxation process observed [24]. Relaxation times obtained in the fitting procedure are collected in Table II.

Interestingly, in dielectric measurements performed during heating of a sample, which had been first cooled rapidly to $123 \mathrm{~K}$, up to room temperature, five relaxation processes were found. First the process " 1 " of a very small dielectric increment was detected. At $153 \mathrm{~K}$, a much stronger relaxation process " 2 " appeared at lower frequency side. Both processes coexist up to $173 \mathrm{~K}$ (Fig. 6), i.e., in the ODIC phase $C 2$, but intensities of both absorption maxima were decreasing with the temperature growth. At $183 \mathrm{~K}$, rotational motions of molecules stopped (see filled triangles in Fig. 7) as 


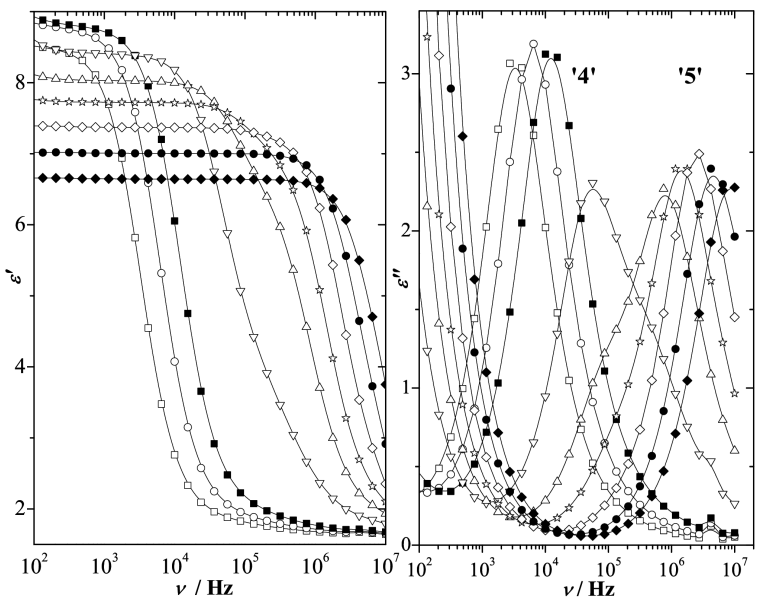

Fig. 8. Dielectric dispersion (left) and loss (right) data for 2,2-DM-1-B observed on heating after fast cooling, showing two relaxations processes: "4" at $213 \mathrm{~K}(\square)$, $218 \mathrm{~K}(\circ), 223 \mathrm{~K}(\mathbf{\bullet})$ and $233 \mathrm{~K}(\nabla)$ and "5" at $238 \mathrm{~K}(\triangle)$, $243 \mathrm{~K}(*), 248 \mathrm{~K}(\diamond), 253 \mathrm{~K}(\bullet)$ and $258 \mathrm{~K}(\diamond)$. The solid lines are the Havriliak-Negami fits. spontaneous crystallization of the ordered crystal $C 3$ occurred. On further heating, in the temperature range 188-208 K, a new process " 3 " of a very small dielectric absorption was observed, growing within the frequency range of $10^{4}-10^{5} \mathrm{~Hz}$ (see Fig. 7). Then, a phase transition to crystal $C$ was observed as a dramatic growth of absorption showing a new slower relaxation process " 4 " $\left(\Delta \varepsilon^{\prime}=0.5\right.$ at $208 \mathrm{~K}$ and $\Delta \varepsilon^{\prime}=7.5$ at $\left.213 \mathrm{~K}\right)$. At about $T_{\mathrm{m} 1}$ (above $223 \mathrm{~K}$ ) process " 4 " transformed to a faster process denoted as " 5 ", which accompanied melting of the rotational phase $C$ (see Fig. 8).

The results of the fits of Eq. (2) to the dispersion and absorption data in phases $C 2, C$, and $I L$ are shown in Figs. 6-8. For the phase $C 2$ the absorption $\varepsilon^{\prime \prime}$ of process " 1 ", in the $\log \nu$ scale has a half-width $W=3.4$, i.e. much larger than $W_{\mathrm{D}}=1.14$, typical for a relaxation of the Debye type [26, 27]. The best-fit values of correlation parameters $(1-\alpha)$ and $\beta$ for motions responsible for relaxation " 2 " do not exceed 0.4. This means a large deviation from the Debye relaxation.

Relaxation time $\tau$ for 2,2-DM-1-B. $\tau$ in $10^{-6} \mathrm{~s}$.

TABLE II

\begin{tabular}{|c|c|c|c|c|c|c|}
\hline \multicolumn{2}{|r|}{$\tau$ on slow cooling } & \multicolumn{2}{|c|}{$\tau$ on heating after slow cooling } & \multicolumn{3}{|c|}{$\tau$ on heating after rapid cooling } \\
\hline$T[\mathrm{~K}]$ & Relaxations "4" and " 5 " & $T[\mathrm{~K}]$ & Relaxations " 4 " and " 5 " & $T[\mathrm{~K}]$ & Relaxation " 2 " & Relaxation "1" \\
\hline 211 & 78.28 & 203 & 258.71 & 123 & & 111546.66 \\
\hline 213 & 60.15 & 213 & 62.01 & 133 & & 15867.54 \\
\hline 215 & 43.57 & 223 & 16.61 & 143 & & 491.54 \\
\hline 223 & 15.97 & 233 & 4.52 & 153 & 2942493.22 & 30.76 \\
\hline 231 & 5.46 & 243 & 1.57 & 155 & 1718412.15 & 16.97 \\
\hline 233 & 3.49 & 253 & 0.07 & 157 & 1034693.79 & 11.12 \\
\hline 235 & 3.49 & 263 & 0.02 & 163 & 228651.56 & 2.39 \\
\hline 243 & 1.21 & & & 173 & & 0.36 \\
\hline 247 & 0.72 & & & & & \\
\hline 249 & 0.28 & & & 213 & & 48.06 \\
\hline 251 & 0.08 & & & 218 & & 24.60 \\
\hline 253 & 0.06 & & & 223 & & 12.91 \\
\hline & & & & 233 & & 2.71 \\
\hline & & & & 238 & & 0.2 \\
\hline & & & & 243 & & 0.11 \\
\hline & & & & 248 & & 0.06 \\
\hline & & & & 253 & & 0.03 \\
\hline
\end{tabular}

The absorption curves of the relaxation processes " 4 " and " 5 " are symmetric in the $\log \nu$ scale and the values of half-width $W$ are close to $W_{\mathrm{D}}$, which means that there is no distribution of the relaxation times. The examples of the best-fit values of the parameters are as follows: $(1-\alpha)=0.98, \beta=1, \tau=(12.9 \pm 1.3) \times 10^{-6} \mathrm{~s}$ for the relaxation " 4 " in the ODIC phase $C$ at $223 \mathrm{~K}$ and $(1-\alpha)=0.83, \beta=1, \tau=(0.11 \pm 0.01) \times 10^{-6} \mathrm{~s}$ for relaxation " 5 " in the liquid phase at $243 \mathrm{~K}$.

Figure 5 presents the temperature dependence of the relaxation rate data, $\log \tau^{-1}(1 / T)$, collected for various relaxation processes observed: for those registered on slow cooling and heating by triangles and circles, respectively, while for relaxation processes " 1 " to " 5 " by squares. Lack of the points in the temperature range of $173 \mathrm{~K}$ to $213 \mathrm{~K}$ means that up to $188 \mathrm{~K}$ motions of the molecules are stopped in the ordered crystalline phase $C 3$ and then no equilibrium phase was established (the non-equilibrium process " 3 " is not presented) till $213 \mathrm{~K}$. One can see that the data obtained during heating of slowly cooled (triangles) and rapidly cooled (squares) samples do not match, meaning that the phase diagram detected 
in each regime of dielectric measurements is different. Moreover, it is worthwhile to say that the rate of the motions detected in ODIC phase $C 1$, which melts at about $250 \mathrm{~K}$, is equal to the reorientation rate in the ODIC phase $C$ melting at about $235 \mathrm{~K}$. In both phases the relaxation is identified as process " 4 " and is of one order of magnitude slower than process " 5 " in the liquid phase. The processes " 1 " and " 2 " in supercooled phase $C 2$ are of theArrhenius type with similar activation energies of $(12.65 \pm 0.2) \mathrm{kJ} / \mathrm{mol}$. The activation energy of process " 4 " in phase $C$ equal to $(14.21 \pm 0.1) \mathrm{kJ} / \mathrm{mol}$ is similar to that of the process " 2 " observed in the phase $C 2$.

\section{Discussion}

Adiabatic calorimetry measurements showed a monotropic system of phases in neohexanol with the phase diagram depending on the rate of cooling of the sample. This was confirmed by the dielectric spectroscopy. However, full consistency of the results of both methods was not achieved. In dielectric measurements on slow cooling and heating of the substance the reversible phase transition was detected: $I L(\approx 251 \mathrm{~K}) \leftrightarrow C 1$ and no supercooling of the liquid phase was registered. No phase transition was detected down to $203 \mathrm{~K}$, but one should keep in mind that in both phases the rates of motions (see Fig. 9) and dielectric permittivities (compare data for phase $C 1$ in Fig. 5 and for phase $C$ in Figs. 8 and 10) are similar.

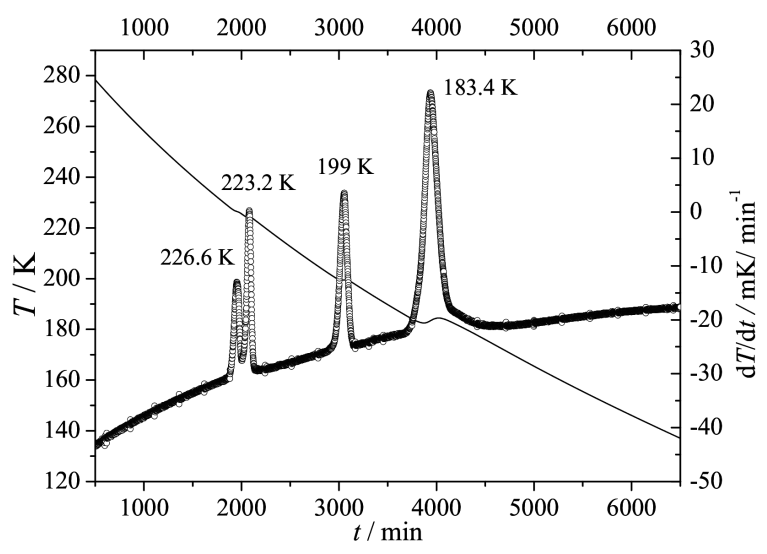

Fig. 9. The drift $\mathrm{d} T / \mathrm{d} t$ vs. $t$ observed on slow cooling. Solid line shows the time dependence of temperature.

Contrary to that on heating after fast cooling, the phase diagram seems to be the following: $C 2$ $(\approx 183 \mathrm{~K}) \rightarrow C 3(188 \div 213 \mathrm{~K}) \rightarrow C(\approx 233 \mathrm{~K}) \rightarrow s I L$. It seems that in the temperature range from $188 \mathrm{~K}$ till about $208 \mathrm{~K}$ kinetics of growth of the rotatory phase $C 2$, as observed by calorimetry, was too slow for $C 3 \rightarrow C 2$ transition to appear. Due to a short time of dielectric measurement at each temperature, thermodynamic conditions became appropriate for activation of rotational motions only above $208 \mathrm{~K}$, when growth of phase $C$ occurred.

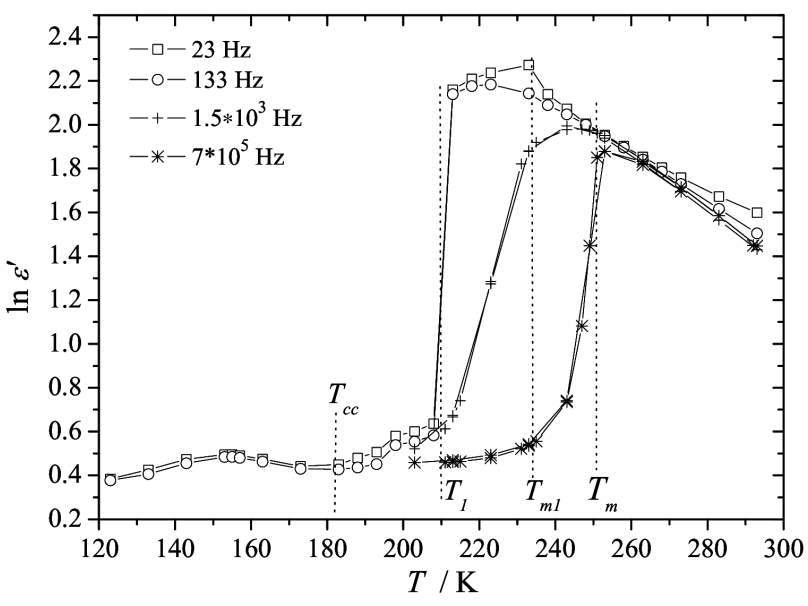

Fig. 10. The real part of the dielectric permittivity $\ln \varepsilon^{\prime}$ vs. temperature observed for heating after fast cooling at $23 \mathrm{~Hz}$ (squares) and $133 \mathrm{~Hz}$ (circles) and during slow cooling and heating at $1500 \mathrm{~Hz}$ (crosses) and $7 \times 10^{5} \mathrm{~Hz}$ (asterisks). The vertical dotted lines show the approximated temperatures of phase transitions.

As an illustration of the phase sequence detected in both regimes of the experiment the temperature dependence of real part of electric permittivity is presented in Fig. 9. The activation of molecular motions at about $T_{1}=213 \mathrm{~K}$ is shown by a jump of the $\ln \varepsilon^{\prime}(T)$ measured on heating after fast cooling (circles and squares were registered at phase $C$ and above $T_{\mathrm{m} 1}$ at liquid phase). The frequencies used were low enough for reorienting molecules to follow the changes of electric field, i.e. to contribute to the orientational polarization measured. On slow cooling/heating one can see a smooth change of $\varepsilon^{\prime}(T)$ for frequency $1.5 \times 10^{3} \mathrm{~Hz}$ (crosses), low enough to see a relaxational feature of electric polarization in ODIC phase $C 1$, while for frequency $7 \times 10^{5} \mathrm{~Hz}$ (stars) a sharp drop/jump at about $T_{\mathrm{m}}=251 \mathrm{~K}$ appears due to the crystallization/melting of phase $C 1$.

In this context it is worth noting that the crystalline phase $C 1$, which melted at higher temperature $T_{\mathrm{m}}$ $[5,15-20]$, seems to be evidenced in the measurement performed using the drift calorimetric method: during slow cooling of the sample with the rate of $-1 \mathrm{~K} / \mathrm{min}$ four thermodynamic anomalies were registered during time evolution of the drift $\mathrm{d} T / \mathrm{d} t$ (see circles in Fig. 9). Simultaneously observed evolution of temperature $T(t)$ down to $120 \mathrm{~K}$ (solid line) allowed us to determine the following values of transition temperatures for the mentioned anomalies: $226.6,223.2,199$, and $183.4 \mathrm{~K}$. They can be ascribed to the following phase transitions: $s I L \rightarrow C 1$, $s I L \rightarrow C, C 1 \rightarrow C 2$ and $s C 2+s C \rightarrow C 3$ (see broadening in the anomaly at $183.4 \mathrm{~K}$ ).

In comparison with the transition temperatures measured by point calorimetric method one can see a significant supercooling of the liquid $(s I L)$ and rotatory phases $(s C 2, s C)$. But when the sample was cooled faster (with $-5 \mathrm{~K} / \mathrm{min}$ ) to $100 \mathrm{~K}$, then only a crystallization of phase 
$C 2$ was registered on $\mathrm{d} T / \mathrm{d} t$ vs. $t$ curve at about $180 \mathrm{~K}$. One can assume that the dielectric relaxations " 1 " and "2" observed up to $180 \mathrm{~K}$ can be related to molecular dynamics in the supercooled ODIC phase $C 2$. It seems that the weak process "1", observed also by Johari [19], can be regarded as the so-called $\beta$-relaxation and related to local motions, while the main relaxation " 2 " as the structural $\alpha$-relaxation connected with reorientations of the whole molecules $[28,29]$. The temperature $140 \mathrm{~K}$, at which molecular relaxation time of the process " 2 " is equal to $100 \mathrm{~s}$, can be treated as the glass transition temperature $[1,28,30]$. This temperature is close to $T_{\mathrm{gII}}$ detected by the adiabatic calorimetry method.

The glass transition in the ODIC phase of neohexanol was registered also by Chan and Johari in dielectric measurements, by Edelmann in DT measurement and by Dworkin in DSC experiment but at higher temperatures. In fact in the drift changes when the sample cooled rapidly to $90 \mathrm{~K}$ was heated very slowly in a regular way (see Fig. 11, $T(t)$ given by a solid line), two glass transition anomalies were visible. In the point method glass-like anomalies manifest themselves by crossing of the "envelope" of the $\mathrm{d} T / \mathrm{d} t$ vs. $t$ (dotted line in Fig. 11) with a zero effective drift line (broken line in Fig. 11) or, in other words, by change of the sign of the $\mathrm{d} T / \mathrm{d} t$ from positive to negative. In this way at temperatures near $T_{\mathrm{gI}}=123 \mathrm{~K}$ and $T_{\mathrm{gII}}=137 \mathrm{~K}$ glass-like anomalies were detected.

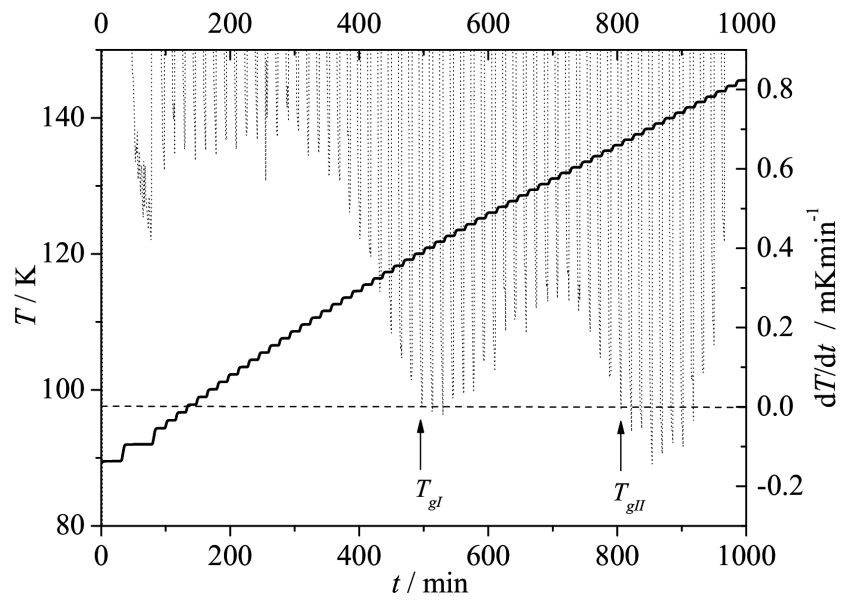

Fig. 11. The spontaneous drift $\mathrm{d} T / \mathrm{d} t$ vs. $t$ (dotted line) for neohexanol in the temperature range of two glass transitions in response to the regular step-by-step heating $T(t)$ (solid line). Broken line corresponds to zero effective drift.

The zero effective drift line was measured earlier for the phase $C 3$ during similar heating, when no phase transition was found up to about $195 \mathrm{~K}$. Thus, the drift method shows that on fast cooling, the phase $C 2$ becomes supercooled, and then vitrified. One can assume that on cooling reorientations of whole molecules are frozen-in at higher temperature while at lower one local motions stop. The huge heat capacity hump accompanying glass transition is related to the formation/destruction of the hydrogen bonds between molecules.

Rich and complicated polymorphism of solid state was found only for 2,2-DM-1-B isomer. The computer density functional theory (DFT) modeling of the 2,2-DM-1-B molecules reveals three rotational conformations (trans, gauche+ and gauche-). This results in different energy minima for various hydrogen bond molecular clusters $[6,7]$. It may cause free energy surface to have many local minima, making the ordered phase $C 3$ difficult to stabilize. The formation of branched and chain aggregates via hydrogen bonding is found widely in crystals (ordered and disordered), with favorable tetramer clusters $[6,7,31]$.

\section{Conclusions}

Polymorphism of solid state of 2,2-dimethylbutan-1-ol was studied in the temperature range from $100 \mathrm{~K}$ to $260 \mathrm{~K}$ using adiabatic calorimetry and from $123 \mathrm{~K}$ to $290 \mathrm{~K}$ using dielectric spectroscopy. A monotropic system of phases was found. Apart from an ordered crystal $C 3$, two ODIC phases $C 2$ and $C$ of $2,2-\mathrm{DM}-1-\mathrm{B}$ were identified using adiabatic calorimetry. No glass of liquid phase was detected. Phase $C 2$ was easily supercooled and vitrified in two subsequent glass transitions. On heating, small anomalies were traced at $T_{\mathrm{gI}}=123 \mathrm{~K}$ and at $T_{\text {gII }}=137 \mathrm{~K}$. Next, metastable phase $C 2$ transformed, through metastable crystalline phases detected also by the earlier DT studies $[15,16]$, to the stable crystal $C 3$ at $180 \mathrm{~K}$. Then, $C 3 \rightarrow C 2$ transition, associated with the largest thermal effect, $C 2 \rightarrow C$ transition and melting of phase $C$ at $T_{\mathrm{m} 1}=234.5 \mathrm{~K}$ were detected.

On heating of the sample cooled rapidly to $123 \mathrm{~K}$, a very weak $\beta$-relaxation process ascribed to local dynamics, and an intensive, slower $\alpha$-relaxation process related to reorientations of the whole molecules was identified by dielectric spectroscopy in the supercooled $C 2$ crystalline. Both relaxation processes are of theArrhenius type and of the similar activation energy equal to about $12 \mathrm{~kJ} /(\mathrm{mol} \mathrm{K})$. Above $183 \mathrm{~K}$, spontaneous crystallization of the phase $C 3$ was observed. Above $208 \mathrm{~K}$, a growth of the ODIC phase $C$ and its melting at $T_{\mathrm{m} 1}$ were detected. Contrary to that, on slow cooling, at about $T_{\mathrm{m}}=251 \mathrm{~K}$ crystallization of the new ODIC phase $C 1$ occurred. On heating, the phase $C 1$ cooled down to $203 \mathrm{~K}$, melted at the same $T_{\mathrm{m}}$ temperature, which corroborated the earlier results of the DT method. Dynamics in the $C 1$ and $C$ phases, melting in different temperatures, is characterized by the same relaxation rates and the same temperature dependence. Relaxation observed in these phases is of one order of magnitude slower than the process detected in the liquid phase. Intensities of the dielectric absorption related to reorientations of whole molecules $(\alpha$-process) in the liquid and in three ODIC phases are of the same order of magnitude. The intensity of dielectric absorption for $\beta$-relaxation connected with local dynamics is of two orders of magnitude smaller. One can 
regard freezing of two-scale dynamics identified in phase $C 2$ as the mechanism of two glass transitions detected in calorimetry measurements.

The details of the phase diagram obtained for neohexanol were sensitive not only to the rate of cooling of the sample but also to the volume of the sample which was different in the two methods used. The phase $C 1$ and its melting were not observed in adiabatic calorimetric experiment despite many efforts. Tendency to glass formation was not found only for the crystalline phase $C 2$. Most of the organic liquids belong to the class of fragile glass formers, whose common feature is the deviation of the $\alpha$-relaxation from the Arrhenius temperature dependence [1]. On the contrary in the ODIC phase $C 2$ of neohexanol the Arrhenius behaviour was found. A slower adaptation of the dipolar molecules to the temperature changes may be caused by the ordering of centres of reorienting molecules in the crystal lattice. Slow softening is typical for the so-called strong glass formers. The same response to temperature changes was found in the orthorhombic crystal-like phase $E$ in some liquid crystals [32]. The temperature dependence of dynamics above $T_{\mathrm{g}}$ (as well as the excess of density of vibrational states in glass) is determined by the details of the free energy surface of the metastable glass-forming thermodynamic phase measured by the fragility parameter $m=\frac{\mathrm{d} \log \tau}{\mathrm{d}\left(T_{\mathrm{g}} / T\right)}[24]$. In case of the ODIC phase $C 2$ of neohexanol a crystal-like ordering of molecules results in $m=16$, meaning a smaller complexity of the surface of free energy than in fragile glass-forming liquids with $m>16$. But the unified theory of fragile/strong pattern of temperature behaviour of dynamics in soft matter is still expected [33].

\section{Acknowledgments}

The authors would like to thank Prof. Antoni Kocot and Dr. Kamil Kamiński for their help in the dielectric measurements at the Silesian University.

\section{References}

[1] A.C. Angell, Science 267, 1924 (1995).

[2] K. Adachi, H. Suga, S. Seki, Bull. Chem. Soc. Jap. 41, 1073 (1968).

[3] M. Sorai, S. Seki, Mol. Cryst. Liq. Cryst. 23, 299 (1973).

[4] S. Urban, B. Gestblom, R. Dabrowski, Phys. Chem. Chem. Phys. 1, 4843 (1999).

[5] E. Juszyńska, M. Massalska-Arodź, P.M. Zieliński, J. Ściesiński, Phase Transit. 79, 899 (2006).

[6] E. Juszyńska, Ph.D. thesis, Institute of $\mathrm{Nu}^{-}$ clear Physics, PAS, Cracow 2008, accessible on: www.ifj.edu.pl/SD/rozprawy_dr/rozpr _ Juszynska.pdf?lang=pl (in Polish).

[7] E. Juszyńska, K. Hołderna-Natkaniec, M. MassalskaArodź, I. Natkaniec, E. Ściesińska, J. Ściesiński, Acta Phys. Pol. A 122, 765 (2012).

[8] M. Gałązka, E. Juszyńska, P.M. Zieliński, Physica B 406, 4342 (2011).
[9] E. Juszyńska, M. Massalska-Arodź, I. Natkaniec, J. Mayer, J. Krawczyk, Physica B 403, 109 (2008).

[10] M. Massalska-Arodź, T. Nakamoto, T. Wasiutyński, J. Mayer, J. Krawczyk, M. Sorai, J. Chem. Thermodyn. 36, 877 (2004).

[11] E. Juszyńska, M. Massalska-Arodź, J. Mayer, I. Natkaniec, J. Krawczyk, P. Tracz, Solid State Phenom. 112, 89 (2006).

[12] E. Juszyńska, K. Hołderna-Natkaniec, M. MassalskaArodź, I. Natkaniec, E. Ściesińska, J. Ściesiński, Acta Phys. Pol. A 113, 1131 (2008).

[13] E. Juszyńska, K. Hołderna-Natkaniec, I. Natkaniec, E. Ściesińska, J. Ściesiński, M. Massalska-Arodź, in: Dynamics investigation of 3,3-dimethyl-2-butanol and 3,3-dimethyl-1-butanol by IINS and FIR methods, Chapter in book: Complementary methods in condensed matter research, Eds. J. Chruściel,A. Szytuła, W. Zając, Wydawnictwo Akademii Podlaskiej, Siedlce 2008, p. 185 (in Polish).

[14] E. Juszyńska, M. Massalska-Arodź, P.M. Zieliński, J. Krawczyk, Acta Phys. Pol. A 122, 693 (2012).

[15] R. Edelmann, Ph.D. Thesis, Ruhr University, Bochum 1990 (in German).

[16] R. Edelmann, U. Bardelmeier,A. Würflinger, J. Chem. Soc. Faraday Trans. 87, 1149 (1991).

[17] A. Dworkin, Faraday Discuss. Chem. Soc. 69, 288 (1980).

[18] R.K. Chan, G.P. Johari, Conf. Electr. Insul. Diel. Phenom., nn. Report 43, 331 (1974).

[19] G.P. Johari, Ann. N.Y. Acad. Sci. 279, 117 (1976).

[20] W. Dannhauser, L.W. Bahe, R.Y. Lin,A.F. Flueckinger, J. Chem. Phys. 43, 257 (1965).

[21] Control and acquisition software written by J. Krawczyk.

[22] Comprehensive Handbook of Calorimetry and Thermal Analysis, Ed. M. Sorai, Wiley, Chichester 2004.

[23] J. Timmermans, J. Phys. Chem. Solids 18, 1 (1961).

[24] W. Haase, S. Wróbel, Relaxation Phenomena, Liquid Crystals, Magnetic Systems, Polymers, High-T $T_{\mathrm{C}}$ Superconductors, Metallic Glasses, Springer-Verlag, Berlin 2003, p. 21 and 78.

[25] S. Havriliak, S. Negami, J. Polym. Sci. 14, 99 (1966).

[26] G.P. Johari, M. Goldstein, J. Chem. Phys. 53, 2372 (1970).

[27] G.P. Johari, M. Goldstein, J. Chem. Phys. 56, 4411 (1971).

[28] E. Donth, The Glass Transition, Springer-Verlag, Berlin 2001.

[29] C. Hansen, F. Stickel, T. Berger, R. Richert, E.W. Fischer, J. Chem. Phys. 107, 1086 (1997).

[30] S. Sastry, Nature 409, 164 (2001).

[31] Y. Suzuki, Y. Yamamura, M. Sumita, S. Yasuzuka, K. Saito, J. Phys. Chem. B 113, 10077 (2009).

[32] M. Jasiurkowska, P.M. Zieliński, M. Massalska-Arodź, Y. Yamamura, K. Saito, J. Phys. Chem. B 115, 12327 (2011).

[33] C.A. Angell, K. Ueno, Nature 462, 45 (2010). 